\title{
PROCESSO DE TRABALHO DO GERENTE DE ENFERMAGEM EM UNIDADE HOSPITALAR - UMA VISÃO DOS ENFERMEIROS
}

\author{
Working Process of Nursing Management at a Hospital Unit - \\ The Nurses' Approach \\ Proceso de Trabajo del Gerente en el Oficio de Enfermeros \\ en Unidad Hospitalar - Una Visión de los Enfermeros
}

VivaneFraniscoSandhes

BárbaraPonpeu Christovem

ZeithRosaSluino

\section{Resumo}

Nestapesquisa delimitames conn objeto deestudbavisão dberfermeroquantoao processo detrabalhodb gerentede

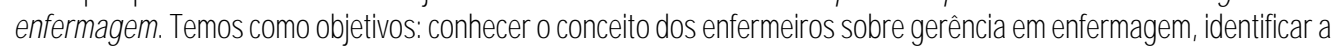

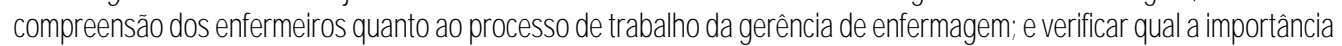
atribuída aotrabalho db gerentedeenfermagem Comm referendial, uilizaramseteorias adhinistratives, conceitos de gerênia. Sua abordageméqualitativa, dotipoestudb decaso, realizadaemumHbspital Uhiversitário nom miápiode

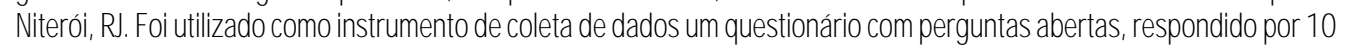
enfermeiras. Os resultados nostramenfermeiras comcorhedimento básico sobre gerênciae o processodetrabalhodb gerentevistoconomitocomplicado.

Palauraschave Efermagem Gerênia Senviços deEfermagem

\begin{abstract}
Resumen

Inthis resesarch wehavedalimitedas object of studythe Enestapesqisaddimitanosconrodjetodeestudiolavisión nurseapproachas norking process of thenursingman- del enfermero aranto al proceso de trabajo del gerente ager. It had as objectives: toknowthenurses conceptas Teniendo como objetivos: conocer el concepto de los to nursing management, to identify the nurses under- efermeros a artoalagerencia, identificar lacomprensión standing as to working process of nursing management; delos enfemeros a arto al procesodetrabajodelagerenda to check what is theimportanceattributed to the work of verificarlaimportancia atribuicad trabajo dl gerente conv nursing manager. As reference, it uas used management referendal, han sidb ubilizadas teorías administratives y theories andmanagemert concepts. Its approadhis quali- conceptos degerencia. Suabordajees a alitativo, del tipo tative, of the casestudy type, doneat a university hospi- estudodecaso, realizadaen un hospita uriversitarioene

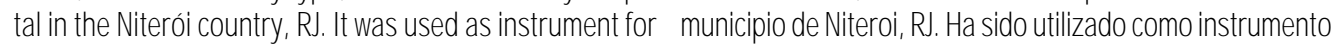
data collecting a questionnare with open questions, an decoledadedadbs unaestionariocon preguntes abiertas, suered bytennurses. Theresults shownurses with besic respondido por diez enfermeras. Los resultadbs mestran knowedgeon managementand the working process of enfemeras con conocimiento básico sobre gerenciay $\mathrm{e}$ manager seenas somethingintricate proceso detrabajodel gerentevisto conmomydfíil.
\end{abstract}

Keymords

Nursing. Managenert NursingSenvices.

\section{Palabasdae:}

Erfermería Cerenda Servicios deErfermería. 
INTRODUÇÃO

Aidéa deste estudb surgiu quandb, ao disatir com colegas na faculdade e no trabalho o fato de gostar e estudar sobre gerênia emenfermagem obsenvaram se mitos comentários conr: "Você estuda gerênaia porque quer mandar, quer ser chefe! Também Gerêndia? Isso não é cargo de dhefia? Já vi que só quer mandar, quer ser enfermesa!"

Náo se pode negar a relação de poder e autoridade que o cargo de gerente transmite Contudb, quanto mais autoridade e poder, maior deve ser a responsa bilidade técrica que este gerente terá, sendo capaz de ser útil ao cresaimento do grupo e da instituição e não apenas ser programado para mandar ou delegar.

A enfermagem ciêndia do cuidar, tem como característica a continuidade, que necessita não só da colaboração de todos os membros da equipe como também dos gerentes, responsáveis por tomar providências para manutenção do senviço de forma favorável. A ação gerendal deve reunir esforços que visemao alcance dos djejivos propostos na prestação da assistênia de enfermagem

Alguns atores referemque a palava gerênia pode ser substituída por administração ou gestão 1; entre tanto, seu emprego depende de quema utiliza, da época emque se está e valor que se confere cada termo ditadb.

Quando se pensa em gerêndia de enfermagem assodiamse as idéas: previsão, aquisição, transporte, recebimento, armazenamento, consenvação, distribuição e controle o que nos recorda o famoso molho de chaves que fica no bolso do enfermero.

A gerêndia emenfermagemque se conhece hoje passou e passa ainda por transformações que resultam em estudos, teorias que fundamentam de forma científica o gerendiar emenfermagem devendo ser capaz de coordenar a assistênia, o potencial humano e os rearsos materiais.

Assim, delimitanos como objeto de estudo a visão do enfermeiro sobre o processo de trabalho do ge rente de enfermagem

Para tal, foramtraçados os seguintes dgjetivos:

- Conhecer o conceito dos enfermeros sobre gerência emenfermagem

- Identificar a compreensão dos enfermeiros quanto ao processo de trabalho da gerêndia de enfermagem

- Verificar qual éa importânda atribuída pelos en fermeiros ao trabalho do gerente de enfermagem
REFERENCIAL TEÓRICO

\section{Conceitos de Gerência/Administração}

Como toda dênia, a administração sofreu influências de vários segmentos durante seu processo de formação: dos filósofos, da lgreja, da organização militar, da revolução industrial, dos economistas liberais, dos pioneiros empreendedores.

Adhinistrar semprefoi una tarefa descaiarte Na atua licade, ta aividade ésubsidiada por dfferentes teorias que permeiamas reformiaçães necessárias à adaptação das constartes instabilidades no nercadb e no mendo.

Cada gerente pode decidr sua estratégia fectur dagnósticos, drmensioner rearsos, planjar sla adicação, resover proddenas, sendbjulgadb pala maneira cono realiza sea trabalho e palos resultados que consegue dos rearsos ds porívis. Logp, não existe una úrica form ceta de umge rerte agir, existem, pois, vérias nanairas de coordener as atividades emuma empresa

A administração evoluiu através de diferentes teorias, e cada teoria valoriza uma ou algumas das seis variáveis básicas da administração: competitividade, pessoas, tecnologia, ambiente, estrutura, tarefas ?

Cada teoria administrativa surgiu como resposta ao problema empresarial de sua época, forambem sucedidas, são aplicáveis na atualidade, e cabe ao gerente conhecêlas para ter a sua disposição emle que maior de altematives?

A gerênda é conceituada como a arte de pensar, julgar, dedidr e agir para doter resultados ${ }^{1}$. Os profissionais graduados emadministração são formados para serem técnicos como emqualquer outra área: enfermagem direito, medicina, entre outras; contudb, para ser gerente há necessidade de desenvolver a capacidade na arte de pensar e julgar para melhor decidir e agir. Odonínio das técricas administratives auxilia o trabalho, porémhabilidades mais complexas permeiama atuação do gerente.

Assim, depreendese que nemtodb adhinistrador tem capadidade gerencial. Nesse serticb, estudos sugeremque perfil db gererte dave estar baseadb emhabilidades/competências para assegurar una prática de qualidade ${ }^{1-2}$.

Compeitividade é una palava que expressa bem o mundo de hoje Traz consigo mudanças, incertezas, exigêndas e gldbalização. Assim para sobreviver neste mundo de constantes transformações, não basta apenes ser umgerente, é preciso ser umbomgerente e possuir características inerentes a esta condição.

Umgerente competente deve ser capaz de mobilizar conhedimentos, informações e até mesmo atitur 
des, pera adicálos, comcapadidade dejulganerto, emsitur accões reais e connetes, indhidulnerte ecomsua equipe detrabalh ${ }^{3}$, reurindb umcorjunto de habilidades.

Existemtrês tipos de habilidades fundamentais pera o gerente 2:

- Habilidade téarica: baseiase emutilizar corhe ámertos, nútodos, técicas e equiparertos para exeação de tarefas, através da experiêndia profissional. Relacionase como fazer, por mio de sua instrucão, experiênaia e educação.

- Habilidade humana caraderizase pela capaddade e pelo discemimento de trabalhar compessoas emequipe Lida coma interação entre pessoas e envolve a capadidade de se comnicar, metiver, coordenar, liderar e solucioner conflitos pessoais ou grupais, visandb cogperação, partidpeção e envilvimento das pessoas.

- Habilidade conceitual: constitu-se na capaddade para lidar comidáias e conceitos abstra tos, e está ligada a pensar, raciocinar, diagnosticar situações e formlar alternatives de solur ção para os problemas. É perceber oportunidades onde ringuémenxerga coisa alguma.

Essas três habilidades requerem competências pessoais distintas, as quais tradizemqualidades de quemé capaz de analisar una situação, apresentar soluções e resolver os assuntos ou problemas, constituindo, assim o maior patrinônio pessoal do admiristrador: seu capital intelectual ${ }^{2}$.

Emumternpo de mulança contínua, adquirir una nova competênia significa abandonar a outra que se tomou uttrapassada Assim, o segredb está na aquisição e desenvolvimerto de competênaias durávés, que são:

- Corhedimento: capadidade de reter e dber continuamente informações, conceitos, idéias, expe riênias eaprendzadb. Deve sempre ser readado pera não se tomer ultrapessada, e dele ser trans formado emcontribuição para a empresa.

- Perspediva: é saber colocar o corhedimento emação, adicar a teoria na prática, ubilizando o conhedimento na análise das situações, nas resoluçães de problena e ra condução da empre sa É a capacidade de pôr emação os conceitos e idéas abstratas, que estão na mente do adhinis trador, tormandb-o capaz de diagnosticar siturções e propor soluções criatives e inovadoras.

- Atitude: é o comportarento pessoal do administrador diante das situações no seu diaza dia, representa seu estilo pessoal que pode fa zer as coisas acontecerem a forma de liderar, motiver, comnicar. Compreende o impulso, a de terminação de inover e a convicção de melhoria contínua, de trabalhar compessoas e fazêlas progredir. Transformando o administrador em umagente de mudanças e inovações.

O gerente que desenvolve as habilidades/compe têndias comb as descritas adima tende a assumir a integralidade do papel de gerente nas categorias: decisónia, informacional e interpessoal ${ }^{2}$.

- Decisória situação ra qua épreciso fazer escolhes, uilizandb informações para sues dedisões, induindb os papés de enpreendinerto, resdução de conflitos, docação de rearsos e negociação.

- Informacional: são ações para manter e de senvolver as informações, induindo os papás de monitoração, disseminação, porta-voz.

- Interpessoal: demonstra como o administra dor interage comas pessoas e influencia seus subordinados, baseando-se emsuas habilidades humanas, induindo os papés: representação, ligação e liderança

\section{Modelos Teóricos de Gerência}

Segundo o modlo taylorista, o gerente privilegia os métodos e as técicas para o aurento da produtividade, sobrepondb o interesse enpresarial ao dos trabalhadbres.

Sob o enfoque fordsta, a função gerendal deve integrar e manter harmonicamente todas as atividades da empresa, sendo constituída de cinco elementos: previsão, organização, comando, coordenação e controle, que formamo processo administrativo e constituemas atribuições do gerente 4

Mais adaptado à época emque viverros, onde o estresse e a incerteza são fatores intrínsecos ao processo de trabalho gerendial, o erfoque contingendial atribui ao gerente: o planejamento, a organização, a direção e o controle, mostrando que é fundamentado emteorias anteriores, porém relativizando os fatos, mostrando que rão há uma única forma de resolver e lidar comuma situação gerendia ${ }^{2}$.

Sob este enfoque, o gerente deve aturar de acordb coma sua experiênaia e a evidêndia dos fatos, e não apenas pela razão, pais o contexto (anbiente) influ na tomada de decisões ${ }^{5}$. A palavra contingênaia significa incerto ou evertud, que pode suceder ou não, dependendo das ciranstânias. A proposição de verclade ou falsidade dos fatos somente será corhedida pela expe riência e pela evidência, e não apenas pela razão.

Neste tipo de abordagem não se alcança a eficá da organizacional segundo umúnico e exdusivo mo- 
delo organizaciona, logo, não existe una forma única e melhor para organizar no sentido de se alcançrem os objetivos variados das organizações dentro de um anbiente tambémvariado. Estudos recertes mostram que a estrutura da organização e seu funcionamento são dependentes de sua relação como ambiente externo. Variações no anbiente ou na tecnologia condur zema variação na estrutura organizacional. Esta teoria é uma relação do tipo "se então" 2 , isto é, se surgemcontingências de maneiras diferentes nas organizações, então há uma variedade de reações estur turais e comportamentais às contingêndias ${ }^{5}$.

O gerente deve considerar que as caradenísticas ambientais condicionamas caradtésticas organizacionais, pais é no anbierte que estão as explicações causais das características organizadionais. Constatada a natureza miltiveriada da organização, verifica-se que as organiza ções operamsob condçães veriáveis e emairanstânias espećficas. Envez de una relação linear de causa e efeito entre veriáves indepondentes do anbierte, há una relação funcional entre elas.

O gerente, sob o enfoque contingendial, utilizase das seguintes práticas administrativas: planejamento, organização, direção e controle, que apresentamos seguintes aspedtos básicos: a organização é de natur reza sistêmica, umsistema aberto; as características organizacionais possurmuma interação entre si e com o meio ambiente; e as características ambientais funcionamcono variáveis independentes, e as caracte ństicas orgarizacionais são variáveis dependentes.

Constata-se que o poder e a autoridade do gerente dependem das injunções contínuas e de informa ções obtidas de form diversificada; ele utiliza a negodação constantemente e se apresenta tenso, nevoso e incerto sobre os resultados de suas decisões. Nâo se reputa ao trabalho do gerente a preponderância da racionalidade, impessoalidade e sistematização das ações, pois a sua atuação está baseada emações desordenadas e intermitentes, com um processo decisónio tambémbaseado emdecisões intuitivas, influenciadas pelo relacionamento interpessoal que se estabdece no dia-a-dia de seu labor ${ }^{1}$.

METODOLOGIA

Esse estudo temabordagemqualitativa, do tipo estudo de caso, realizado emumhospital universitá no localizado no muriápio de Nterái-Rl. O projeto de pesquisa foi aprovado pelo Comitê de Ética emPesquisa da Instituição. Foi garantido o anonimato dos sujeitos, os quais aceitaramparticipar do estudo, as sinando o Termo de Consentimento Live e Esdared- do, e foramobedecidos todos os demais requisitos éticos consignados na Resolução ONS n196/96.

Essa pesquisa foi realizada como produto final do Curso de Especialização em Cerênia dos Serviços de Enfermagemna Universidade Federal Fuminense.

Os sujeitos da pesquisa foram 10 enfermeras que atuamno Hbspital Universitário, vinaladas às nove ge rências de Enfermagemque compõema Diretoria de Evfermagemda referica instituição. Os citérios de indur são dos sujetos foram trabalhar no hospital universitánio darianete e ser responsável pelo trabalho de enferma gemde algun a unidade de internação.

Ac deta de dados fá redizada no nếs de maio de 2005, sendb uilizadb u mquestionánio contendo perguntas doetas.

Os dados foramtrabalhados por meio de análise temática ${ }^{6}$ e apresentados emtrês categorias: conceito dos enfermeiros sobre gerência em enfermagem conhecimento sobre o processo de trabalho da gerênaia de enfermagem e a importância do trabalho do gerente de enfermagem as quais foramdisatidas combase emliteratura pertinente

APRESENTAÇÃO E DISCUSSÃO DOS RESULTADOS

\section{Contextualizando o Cenário}

O hospital universitário, entre os mais de 6.000 hospitais do país, faz parte do Sisterra Útico de Saút de (SUS), integrando os 154 que foramreconhedidos pelos Mnistérios da Saúde e da Educação como de ensino ou auxiliares de ensino?.

Nessa condição, em2001, foi co-responsável por aproximadamente $9 \%$ dos leitos, $12 \%$ das internações e 24\% dos rearsos do SUS destinados ao paganento de internações. Sua característica de atendimento é direcionada para a rédia e alta complexidade. Assim por seu caráter de referênda, concentra os pacientes mais graves, que exigemmis rearsos e atenção.

Tal posição corresponde ao papel assistencial proposto para esse tipo de hospital pelo próprio Conselho Nacional de Saúde, que considera essendia sua concentração mo atendimento emníveis terciánio e quatemánio, abrangendo ações de rédia e alta complevidade.

A par deste destacado e bemdefinido papd, a enfermagem tem destaque preponderante para a conseação dos dojeitivos da instituição.

$\mathrm{Nb}$ deserho organizacional, a equipe de enfermagemdo hospital universitário é subordinada à Dretoria de Enfermagem(DE/HU), a qual é diretamente ligada ao Dretor Geral do hospita.

A DE/HU possui nove chefias, denominadas gerências: Emergência; Internação e Ata, e Ambulatório; Cínica Espedializada; Neonatologia e Pediatria; Enfermagemđínica; Obstetría; Imageme 
Diagnóstico; Centro Grúrgico e Central de Material; EnfermagemCinúrgica. Cada gerência é responsável pela coordenação de vérias u vidades, tanto emrelação à assistência quanto aos reaursos humanos, dotadas de capacidade de decisão segundo a filosofia e dojetivos da DE/HU

Integram tanbéma DE/HU a Coordenação do Programa de Educação Permanente de Enfermagem e a Equipe de Supenvisores. Essa equipe responde pela DE/HU mo horánio noturmo e nos finais de serana.

Conceito dos enfermeiros sobre gerência em enfermagem

As técricas administratives são entendidas como definições daras sobre planos, padronizações, rotinas, atribuições funcionais que condicionamcomportamentos, fazendo do administrador umplanejador sistenático e umcoordenador e supenvisor eficiente das atividades organizacionais's.

Indúdos nas técicas administratives estão os instrumentos, os quais subsidiama ação administrativa, favorecendo o acesso às informaçōes necessánias à tomada de decisão, tanto quanto for possível, ressaltado o aprimoramento da sua forma, construção e reavaliação, a fimde atualizálos para que sejamade quadamente ubilizados ${ }^{\text {. }}$.

Neste sentido, algumas das enfermeras, quando indagadas como conceituariamgerêndia emerfermagem reladionaramas técricas administrativas à ação gerenial desenvolvida pelas gerentes de enfermagem

Processo de, au conjunto de processos pelos quais se viabiliza a organização hospitalar ou setorial, visando pronover, de forma hamônica, o desenvolvimento des atividades emenfermagem (DI)

É [...] una dênda que retrata a parte adninistrativa emrelação à equipe de erfernageme à assistêna ao paderte emu ma uidadehospitdar. (DB)

O gerente emenfermagemé umadministrador da assistência de enfermagem comatividades voltadas não só para a admissão de funcioná rios e escala de trabalho, mas tambémpara o bemestar no local de seniço, comcondições favoráveis à exeação das atividades e ao ber estar dos padientes té a sua alta. (D5)

É o processo de drecionar e atribir atividades elau funções dertro do conteto de trdballo, descde a pre visãa, provisão de mateiais téa pronoção da qu licadeassistendal deerfermagem (D9)

Enfermagemadministrativa, ou seja, aquela que organiza as equipes, providenda o suprimento de materiais do setor, assimcomo a mantenção da aparelhagem É tambémquemfaz a ligação entre os plantões, cominformações pessadas. (D10)

Obsenva-se que a idéa de previsão, provisão, amazenamento de materiais e controle está enraizada nos pensamentos de quase todas as enfermeiras que participaramdo estudo quando o assunto é gerênaia em enfernagem Contudo, destaca-se uma preocupação crescente, e presente em alguns depoimentos, na proara da qualidade na assistência de enfermagem ainda que discreta.

Otras fizeramo vínalo direto como cargo de dhefia caupado pelas gerentes de enfermagem

Entendo que gerênaia emenfermagemé seme Ihanteà dhefia de senviço. (D4)

É uma nomendatura atud, seria o líder emum cargo de chefia (DB)

Drateas ertreistas, algunæs depoentes revelaramnẽo ter intimidade como termo gerênda emenfermagem dfia ldade esta vencida por algu mæs e não por a tras Apesar desta dbsenvação por parte de algumas enfermeras, as participartes do estudb verbalizaramsues conceppõoes sobre o termo gerênaia de enfermagem

Conhecimento sobre o processo de trabalho da gerência de enfermagem

Qando indagadas Sobre o que você pode dizer sobre conve é constituído o processo de trabalho da ge rência de enfermagem?, obtivernos os seguintes de poimentos das enfermeiras:

Depeenderá do tipo de orgarização addada, næs de pende da distribuição detarefas. Ensetratando de efernagem, o trabalho engerênja constitirse đra vés de una visão gerd de setares ou das pates em busca de ofereer, ra redda do possíd, supate pra solucionar intercorênias e auxiliar ra organização estuturd setoria. (DI)

Oprocesso de trabalho da gerênaia de erfermagem dever ser constitúdo pela aveliação do enfermerro. Dlagnósticos das árees de atueção de enfermagem pera implemetar a equipe adequeda (DD)

Oprocesso da gerênaia emenfermagemdaver estar centrado nos aidados das necessidades básices do homem, favorecendo u mequillbrio encoiond entre as equipes de enfermagem comvelorização desues queixes, opiniões eexpriênias. (D5)

Planjamento, coordenaçãa, prover nateriais: reaursos mateiais e hu manos para que as atividades sejam desemperhadas. Pronover atividades ed catives para que as etapas e tareas desemperhadas terhamum padrão. Pronvever ações qualitaives para nanter a qulidadeassistendid. (D6) 
Direciona as ações de enfermagem planeja, exe ata, supenvisiona, é o grande orientador que mantéma equipe comcoesão. (D8)

É constitúdo descle a previsão, provisão de materiais até a busca para a promoção da qualidade assistendal de enfermagem (D9)

Com base no enfoque das teorias dássica e contingendial, o processo de trabalho administrativo é constituído por atribuições comns comb: planejamento, organização, dreção e controle; apesar de possut íremênfases diferentes. Percebese pelos depoimentos que a base utilizada para as respostas é pautada no modlo dássico de administração, já erraizado nas enfermeiras. Para Teixeira', administrar deve ser entendido comb umesforço organizado para realização de tarefas, emtodas as árees e emtodos os níveis da organização e hierarquia, de forma que todos os dbjetivos propostos sejamalcançadbs, que, neste caso, é a própria assistência emenfermagem Obsenverse, combase nos depoimentos e no autor citado, que a êrfase nas tarefas predonina e os prinápios da adhinistração dentífica também

Somente una enfermera ditou o diagnóstico como meio para atuação do gerente de enfermagem diagnóstico este que permite, independentemente da teonia de preferênia do gerente, partir de una realidade para a implementação de umconjunto de ações efica zes, ou seja, realizar o planejamento.

Oprocesso de trabalho gerendid ettenddb cono cons titúdb de habilidades mais complexes que a de u madhit ristrador não apareceu emnerhuma das respostas.

Unta depoente respondeu que não entendia como é constituído o processo de trabalho do gerente; no entanto, entenderros que o processo de trabalho da gerência ocorre emqualquer nível hierárquico da organização-enfernagem pois as habilidades evigidas facilitamo gerenciamento da assistêndia ao diente, ou seja, o gerenciamento do aidado. Afinal, o enfermeiro deve ser capaz de mobilizar os seus conhedmentos e informações para aplicálos, comcapadida de de julgamento, emsituações reais e condretas, individualmente e com sua equipe de trabalho no direcionamento da assistência prestada, reunindo, assim umconjunto de habilidades.

Das enfermeras responderamsobre o processo de trabalho, porém fazendo referência ao deserho organizacional da enfermagemno hospital emestudb:

Constitúcb por enfermiros(as), quefazempatedeuna dretaria e ca adhinistração hospitalar. (DB)

Corcdara o trabdlo desendvicb res seyões sobsuares pansablilibadee édo entreas seções ea dretoria (D4)

\section{A importância do trabalho do gerente de enfermagem}

Quando questionadas sobre a importância do trabalho do gerente de enfermagem obtiverros as segintes respostas:

Se o gerente 'funiona', au realiza suas tarefas harmonizando os rearsos humanos e materiais a sua importânaia na instituição é ampla. (DI)

Responsável pela área de atuação de enfermagem supervisão das equipes, recntamento e seleção de pessoas, adhinistração hospitalar, bemcomo todas as atividades de educação continueda (D2)

Verificar se os dributos da assistênia emsi etão sendb colocadbs emprática, redizar compra de nateriais para uso hospitala, manter o controle de pessoal quarto a férizs, licença, atributos que possamvir a ocorrer como pessod caerfermagemetc (DB)

Agilizar a comnicação e resoluções entre as se çôes e a diretoria (D4)

Otrabalho do gerente de enfermagemtema importância da dinânica da assistência de enfermagemque viabiliza todos os aidadbs para nanter a equipe de enfermagemunida e influenciando ra qualidade da assistênaia do paciette (DБ)

A gerênia faz coordenação de senviços, usa una pa dronização e qualificação da assistência Permite avaliar e reaveliar as açōes, visando sempre a qualidade da assistênda e prinapalmente una visão macro no sisterna desaúde (D6)

Temqueter alguémcoordanand, alguémno gupo que dêcrientaçãoémito inpartate (DB)

Aimportâna éfundanertd, prinipanmente quento à ogarização elomandanerto dos platões. (D10)

Obsenva-se uma preocupação como bomanda mento dos plantões, a padronização da assistênaia dos senviços de enfermageme certa díficuldade para re latar a importância do trabalho do gerente.

São erfatizadas a supervisão e a coordlnação con partes importantes do processo de trabalho do ge rente, pois a supenisão é umprocesso educativo e contínuo, que consiste, fundamentalmente, emmotivar e orientar os supenvisionados na execução das atividades combase emnormas, a fimde manter de veda a qualidade dos senviços prestados. Já a coorde nação temcomo finalidade harmonizar todos os atos de uma instituição, buscando seu sucesso, equilibrando rearsos físicos, materiais e humano.

Quando as enfermeras falamsobre a importância do trabalho do gerente de enfermagem o fazemca- 
raderizando o processo de trabalho. Consideramcada etapa importante para a assistênda cono umtodo.

Contudo, uma enfermera atingiu o ponto-chave sobre a importância do trabalho gerencial:

Éfundanerta. Sea gerênia rẽo fơ adequadạ, podese ter tocka estrturafísica, operaciond, equipe e assistên ciade erfermagencompronetica (D9)

\section{CONSIDERAÇÕES FINAIS}

As enfermeiras possuem conhedimento solore a adhinistração emerfertagem apesar de seus conceitos rẽo serembeseados formælnerte emteorizs adhiristratives.

A literatura que embasou esse estudb, buscando diferenciar/aproximar o trabalho administrativo do gerencial, nos mostra que o conhedimento das enfermeiras ainda é tímido, pequeno, diante da complexidade do processo de trabalho gerencial. Contudo, apontamuma expressiva preocupação com os resultados da gerênaia no que óz respeito à qualidade ca assistênda de enfermagem, enfocandb a supenisão ea coordeneção pera atingir esse djetino. Patindb cla previssa de que corhecer não transforma, há a necessidade de se compreender, não podenros visluntorar grandes transformaçōes no processo de trabdho gerendal já que as depoertes rẽo o compreendem.

\section{Referências}

1 MuttaPR Cestãocontenporânea adîniaeaatedeser dingente 5ạed. RiodeJaneiro(R)): Record, 2002.

2. Criaventol. Introd çãoàteoriagerd daadhiristração: unavisão

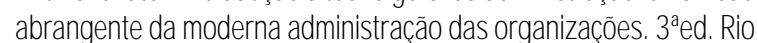
deJaneiro(R): : Esevier, 2004.

3. Conselho Nbiconal deEducação. Parecer n? 16 de 05 deatubro de 1999. Diretrizes Currialares Nacionais para a Educação Profissiond deNvel Técrico.

4. Fayol H Adhinistração industrial egera: previsão, organizacãa, conandb, coorderaçãoecontrde 10"ed SãoPaulo(S): Atlas; 1989.

5. Slvimo $\not R$ Desafios do erfermeiro no desemperho da função gerenial nohospital uriversitáio: una arálisecontingendal [tesede professor titular]. Nterói (BJ): Escola deEnfermagemAurora de Afonso Costa/UF;2006.

6. BardinL Análisedeconteúdb. Lisboa(PT): Ed70; 1979.

7. Portaria Interministerial MEQMS no 1.005, de 27 de maio de 2004. Defineos dba mentos pera verificação do a mprimento dos requisitos dorigatónios relacionadbs aos inisos l a XMl dbartigo $\wp^{\circ}$ da Portaria Interministerial MECIMS r 1 1.000, de 15 de abril de 2004. Dário Ciicial da Reaublica Federativado Brasil, Braslia(DF), 31 maio 2004: Seção 1:54.

8. ParraMT, Mdo MRACAções adhinistratives doenfermerrofrente ao absentésmo. EscAmaNkryRevEferm2004abr, 8(1): 29-38.

9. TexieraEA Teariagerd da adhinistraçãoeprática TCASP. Riode Janero(P): $\mathrm{FG}$, 2003.
O processo de trabalho da gerênia emerferma gemparece ser ainda una incógnita. Apesar de a finalidade, segundo a maioria das enfermeiras, ser a mesma, prestação dos senviços de enfermagem não há umúnico meio de gerendiar.

Todo corpo predisa de una cabeça, eis a idéa re tratada na importância do trabalho do gerente de enfermagem alguémresponsável emagilizar a comnicação, tomar dedisões, coordenar o senviço de enfermagempara qualificar a assistênda prestada, como objetivo de tomála eficiente e eficaz.

A enfermagem diênia do auidar, está emconstante movimento, assimcono toda dênda, comseus pesquisadores e estudiosos que buscamincansavelmente repostas aos seus anseios, superando barreiras e vencendo difia ldades.

Gerenciar depende nais do que boa vontade e um inocente desejo de querer ajudar. É preciso ter sensibilidade para como diente e recorhecer no outro um potencial humano dheio de mistérios e umtesouro de conhedimentos e experiências inesgotáveis. É perce ber que, de sua capacidade de gerenciar, o melhor poderá ser féto ou não.

\section{Sobre as Autoras}

\section{VianefraniscoSanches}

Enfermera Especialistaem Cerêniados Senviços deE Efertagem MentbrodbNídeodeEstudosePesquisas emadadariae Cerência naEfermagem(NECG da da Escolade EnfermagemAurorade Afonso Costada UniversidadeFederd Huminense( $\boxplus A A C U F)$.

\section{Búb:aPonpeuChistoam}

Evermeira VceCoorderadbradbNEQGENdaEAACUF. Docente daÁrea deAdhinistração em Enfermagemdo Departamento de Fundanertos de Enfermageme Administração. Mestre em Efermagempela压田.

\section{ZerithPosStio}

Efermera DretoradeE fermagendoHospital UniversitáioAntônio Pedro/UF. Coordenadbra do NEQGEN Datora emErfermagem

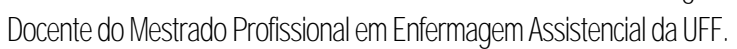

